\title{
Literature Review of Interventions for Between-Task Transitioning for Individuals with Intellectual and Developmental Disabilities Including Autism Spectrum Disorders
}

\author{
Christopher A. Tullis • Helen I. Cannella-Malone • \\ Daniel O. Payne
}

Received: 12 August 2014 / Accepted: 15 October 2014 / Published online: 28 October 2014

(C) Springer Science+Business Media New York 2014

\begin{abstract}
Transitioning between activities or settings may pose great difficulty for individuals with intellectual and developmental disabilities, yet engaging in independent, smooth transitions may be a requisite skill for inclusion in a number of school and community settings. In the current empirical literature review, 32 studies that focused on teaching independent transitioning skills or decreasing challenging behavior in the context of transitions were examined. Studies were divided into two general categories: (a) decreasing challenging behavior and (b) teaching independent transitions. Each general category was then divided into subcategories: antecedent- or consequence-based strategies. The goals of this review were to determine what interventions are most prevalent and effective in the current literature base, identify the extent to which effects are maintained and generalized, and suggest directions for future research.
\end{abstract}

Keywords Transition skills · Between-task transition . Developmental disability $\cdot$ Intellectual disability

Autism spectrum disorders

Students with a variety of intellectual and developmental disabilities (IDD) may experience difficulties engaging in transition behavior (Arlin 1979), defined as a teacher or other environmental prompt to cease an ongoing activity or task and begin to engage in another task (Arlin 1979; Newman et al.

\section{A. Tullis $(\square)$}

Department of Educational Psychology, Special Education, and Communication Disorders, Georgia State University, PO Box 3965, Atlanta, GA 30302, USA

e-mail: ctullis2@gsu.edu

H. I. Cannella-Malone $\cdot$ D. O. Payne

The Ohio State University, Columbus, OH, USA
1995). An example of such transitions is when preschool teachers instruct students to put their free play toys away and proceed to a group area for instruction or a group activity. Estimates have placed the percentage of time students may be expected to transition in some educational settings (e.g., early childhood settings) at approximately 25 to $30 \%$ of the day (Carta et al. 1990; Schmidt et al. 2000). For some students, transitioning from activity to activity may be fairly innocuous. In contrast, some people with IDD including autism spectrum disorders (ASD) may have great difficulty engaging independently in transitioning (Flannery and Horner 1994).

The reasons for transition difficulties (or lack of proficiency in transitioning independently) may be due to two main deficits. First, students may engage in challenging behavior such as self-injury (McCord et al. 2001), noncompliance (Banda and Kubina 2006), or other disruptive behavior (Flannery and Horner 1994). The function of these types of challenging behavior may be to escape the demand placed by requiring a transition or access to the activity that has been terminated (Davis et al. 1998). Second, students may lack the skills to transition independently from activity to activity or their transition behavior may be dependent on teacher or caregiver prompts (MacDuff et al. 1993). These challenges with transitions may limit a student's access to community activities and social interaction and could result in a more restrictive educational or vocational placement (Newman et al. 1995; Schreibman et al. 2000). Additionally, transitionrelated challenging behavior may disrupt classroom routines because the teacher's attention may be shifted from the whole class to one student or group of students (Sterling-Turner and Jordan 2007).

In contrast to individuals who engage in challenging behavior around transitions, individuals who transition independently may have routine access to a much wider variety of 
environments and activities (Sterling-Turner and Jordan 2007). Moreover, success in these environments may be at least in part aided by independently transitioning from activity to activity (Rule et al. 1990). For example, if an individual independently and smoothly transitions from vocational activity to vocational activity, the probability of success in, or at least access to, a typical vocational setting may be elevated.

A growing literature base now exists related to teaching transition behaviors and formulating interventions for transition difficulties. Despite this growing body of literature, several questions may remain in relation to the effectiveness of specific types of interventions (i.e., antecedent- and consequence-based interventions), maintenance and generality of effects, types of implementers, and the use of preassessment technology. Additionally, a thorough review of current research-based practices is absent, which may prevent a clear view of what is currently known about transitionrelated difficulties with people with IDD and/or ASD. Further information related to these areas may allow practitioners to more accurately assess what interventions are the most efficient and appropriate to use given a specific context. To address these areas, the goal of this literature review was to answer the following research questions: (a) What interventions are the most prevalent in the research literature? (b) What interventions are the most effective for addressing challenging behavior in the context of transitioning from activity to activity? (c) What methods are the most effective for teaching independent transitioning to individuals who do not engage in challenging behavior during transitions? (d) To what extent are the observed effects maintained or generalized? and (e) Where are interventions taking place, and who is implementing them?

\section{Method}

\section{Inclusion Criteria}

For the purpose of this review, transitioning was defined as teacher-, supervisor-, or trainer-directed shifts in activities where the students or workers were expected to terminate their ongoing activity and shift their attention to a different activity (Arlin 1979). Studies were included in this review based on the following criteria: each study (a) was an assessment or intervention study concerned with either teaching transition skills or addressing challenging behavior that occurred in the context of transitioning, (b) implemented a single-subject research design, (c) had at least one participant with a reported intellectual or developmental disability (e.g., ASD), (d) utilized direct observation to collect data, and (d) was reported in a peer-reviewed, English language journal. Only data from the participants with a disability were included in the studies in which some, but not all participants were diagnosed with ASD or other IDD. If these data could not be disaggregated, the study was excluded. Additionally, articles that reported solely anecdotal records, dissertations, master's theses, and literature reviews were excluded. For example, although Wilder et al. (2006) conducted functional analyses and implemented an intervention for two typically developing children who engaged in tantrum behavior associated with transitions, it was excluded because neither individual had an IDD.

\section{Databases Searched and Data Collection}

Electronic searches were conducted using ERIC, PsycINFO, MedLine, Web of Science, Electronic Journal Center, Education Research Complete, Education Full Text, Social Sciences Abstracts, and Google Scholar without a date constraint because a previous systematic review had not been conducted. The following terms were searched systematically: (a) transition, (b) between-task transition, (c) developmental disability, (d) latency to transition, (e) intellectual disability, (f) transition-related difficulty, $(\mathrm{g})$ teaching transition-related behavior, (h) autism spectrum disorder, and (i) on-schedule/ontask behavior. Following the electronic search, the reference lists of all identified articles were hand-searched to identify a more complete set of articles.

Data were collected on each study using a structured data sheet that included the reference; sample size; age, gender, and diagnoses of included participants; setting; type of implementer; results of a functional behavior assessment (i.e., function) if conducted; intervention type (i.e., specific intervention and if it was antecedent- or consequence-based); experimental design; and whether measures of generalization or maintenance were collected and the results of those conditions. Data on generalization were collected on the type of generalization (e.g., setting), and data on maintenance were collected on the time period maintenance was collected (e.g., five sessions, 1 year). Antecedent-based interventions were defined as procedures that manipulated stimuli independent of the maintaining contingency (Cooper et al. 2009). Consequence-based strategies were defined as interventions that manipulated the availability of reinforcement or punishment contingent upon the occurrence of a target response (e.g., differential reinforcement). Results of the studies were classified as positive, negative, or mixed based on the determination of the authors. Positive effects were noted when the authors indicated that the procedure was effective for all included participants (e.g., Spriggs et al. 2007). Negative results would have been indicated if the authors stated that the intervention did not result in an effect for any of the participants included; these results were not located in the current literature search. Mixed results would have been noted if the authors stated that the intervention was effective for some, but not all of the participants; these results were not located in the current literature search. 
In addition to qualitative measures, percentage of nonoverlapping data (PND; Scruggs et al. 1987) was calculated for the interventions in each study. This method allows for a metric to be calculated, quantifying the effects of single-subject research. Using PND, studies may be classified as having strong effects (PND greater than $70 \%$ ), questionable effects (PND between 50 and $70 \%$ ), or no effect (PND less than 50; Scruggs and Mastropieri 1998). For studies where the expected outcome of intervention was an increase in the target behavior, the highest point observed in baseline was identified, and a line parallel to the $x$-axis was drawn through this point across intervention conditions. All intervention data points that fell below this line were counted and subtracted from the total number of intervention points. The resulting statistic was divided by the total to yield a percentage of points that fell above the drawn line and did not overlap with baseline. In the studies where the expected outcome was a decrease in the target behavior, the same procedure was used, except the line drawn across conditions passed through the lowest point in baseline and all points above the line were counted and subtracted from the total.

When calculating PND, procedures similar to those outlined by Scruggs et al. (1987) were followed. In order to account for some limitations of PND and to ensure data were reported on the most effective interventions when comparisons were studied, the following modifications were made to the procedures of Scruggs et al. (1987). First, if two interventions were compared or if an alternating treatment design was implemented, the PND for the most effective intervention was calculated for each participant and an average across participants was calculated for each intervention (e.g., Cihak 2011). Only the PND for the most effective intervention was reported because the goal of the current investigation was to identify the most effective interventions for teaching transition behavior and decreasing transition-related challenging behavior. Second, maintenance, generalization, or other follow-up data were excluded from the PND analysis, as the focus of the PND calculation was on the intervention (e.g., Cihak et al. 2010). Third, PND was only calculated for data that were directly related to transition behavior (e.g., Mechling and Savidge 2010). Fourth, if a ceiling was created by extreme data in baseline (e.g., 0 or $100 \%$ ), these data were excluded and an equal number of data points that occurred immediately after the phase change was excluded in the intervention phases (Didden et al. 1997). These data were excluded because the occurrence of data that was at these extremes would automatically yield a PND of $0 \%$ indicating no effect, when an effect was actually present (Didden et al. 1997).

\section{Interobserver Agreement (IOA)}

A second reader independently scored $32 \%$ of the articles selected for inclusion in the current literature review. The reader was given the same data collection sheet and provided with an explanation of each section that was to be scored. An agreement was scored for each study if the primary and secondary reader noted the same type of intervention (antecedent- or consequence-based); participant number, gender, age, and diagnoses; setting; implementer; experimental design; functional behavior assessment (FBA) results; intervention type; results; generalization; maintenance; author reported outcomes (e.g., positive); and PND. The total number of agreements was summed and divided by the total number of articles with IOA, then multiplied by 100 to yield a percentage. All articles were scored with $100 \%$ agreement.

\section{Results}

Tables 1 and 2 provide an overview of data collected for antecedent- and consequence-based methodologies across challenging behavior (Table 1) and teaching transitionrelated behavior (Table 2). Studies in this review were divided into two general categories based on research question (i.e., decreasing challenging behavior or teaching transition-related skills) and then subdivided into two categories based on intervention type (i.e., antecedent- or consequence-based). Thirty-two studies met the inclusion criteria.

\section{General Findings}

The findings from this literature review are presented in Tables 1 and 2. In the current review, studies included 91 total participants ranging in age from 3 to 38 years. Individuals with an ASD (PDD-NOS and Asperger's included) diagnosis alone were the most represented group of participants, accounting for $41 \%$ of the participants. Individuals with moderate to severe intellectual disability without another diagnosis accounted for $11 \%$ of the participants, and $17 \%$ of the participants were individuals with a diagnosis of mild to moderate intellectual disability alone. Participants with ASD and a secondary diagnosis (e.g., mild intellectual disability) comprised $7 \%$ of the total participants included in the current review.

Classrooms were the context for intervention and teaching in $63 \%$ of the studies, and worksites comprised $13 \%$ of the studies. Group homes or hospital settings comprised $13 \%$ of the studies. The remaining $6 \%$ of the studies were conducted in community settings. Teachers or vocational supervisors were the interventionists in $53 \%$ of the studies. Maintenance of treatment gains was collected in $43 \%$ of the studies. Measures of generalization to novel environmental stimuli (e.g., instructors, settings) were collected in $28 \%$ of the studies. 


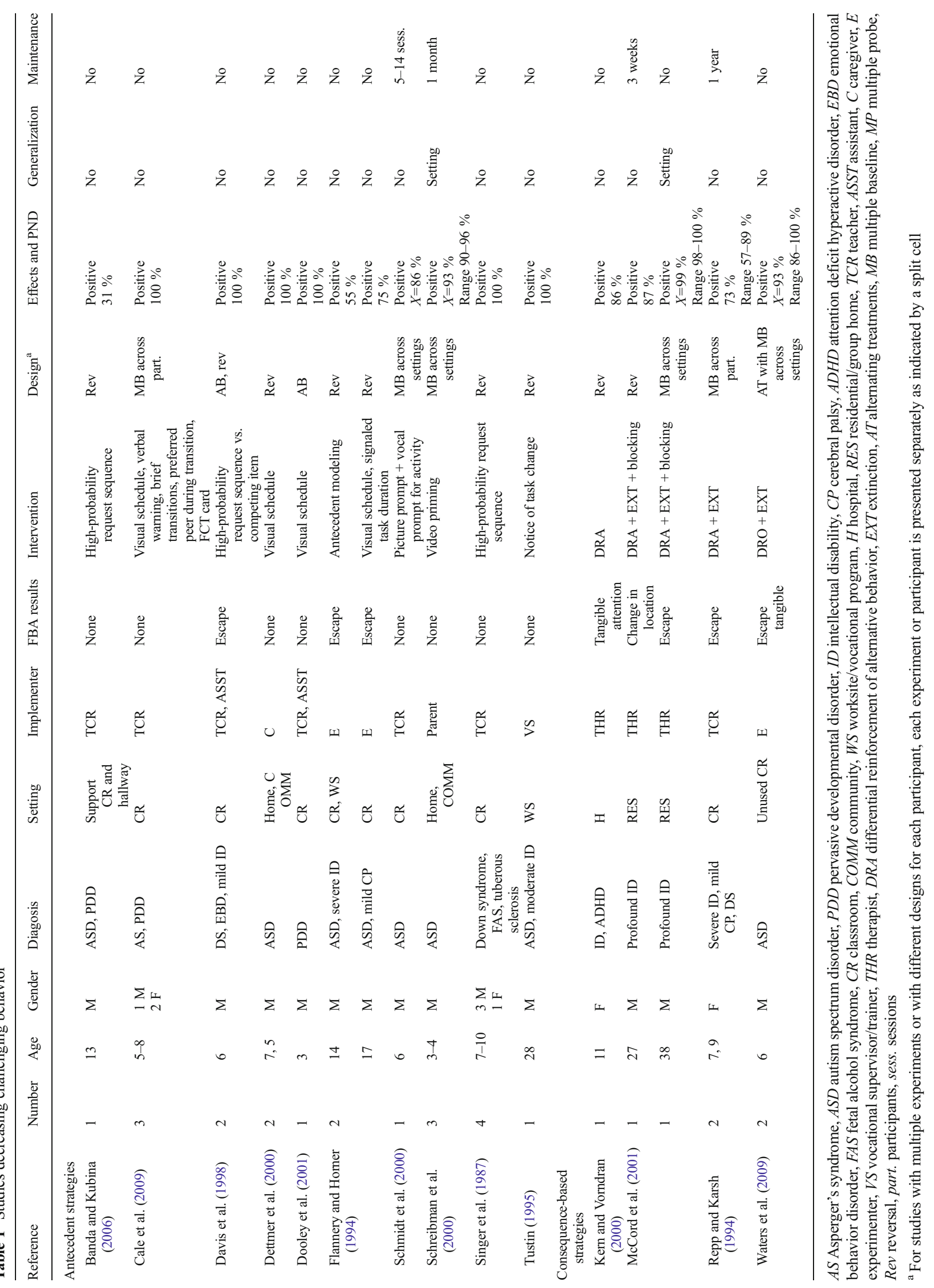




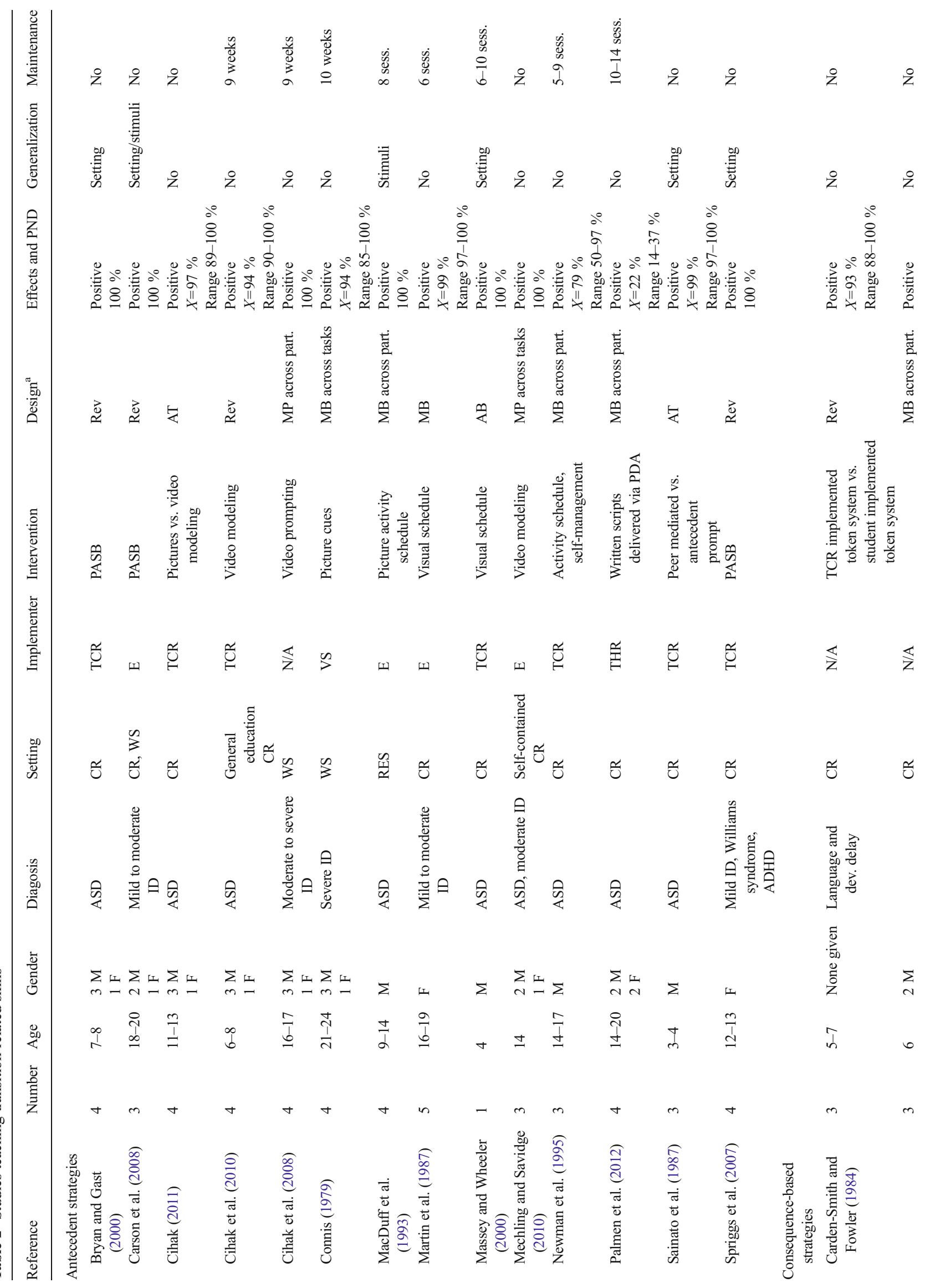


When challenging behavior was targeted for reduction, antecedent-based interventions were implemented in $71 \%$ of the studies and consequence-based interventions were implemented in $29 \%$ of the studies. In those studies, functional analyses or functional behavior assessments were conducted in $43 \%$ of the studies. A slight difference was observed between the PND for antecedent-based and consequencebased interventions. The average PND for antecedent-based interventions was $94 \%$ (range 31-100\%), and the average PND for consequence-based interventions was $91 \%$ (range 86-100\%).

When teaching independent transitioning skills was targeted without the occurrence of challenging behavior, antecedent-based strategies were implemented in $78 \%$ of the studies and consequence-based strategies were implemented in $21 \%$ of the studies. A large difference in PND was observed between antecedent- and consequence-based strategies for teaching independent transitioning behavior. An average PND of $92 \%$ (range 22-100\%) was found for antecedent teaching procedures. In contrast, an average PND of $83 \%$ (range $65-100 \%$ ) was found for consequence-based strategies.

\section{Overview of the Studies}

Antecedent-Based Strategies for Challenging Behavior

Ten studies were identified that addressed challenging behavior in the context of transitioning using an antecedent-based intervention (Banda and Kubina 2006; Cale et al. 2009; Davis et al. 1998; Dettmer et al. 2000; Dooley et al. 2001; Flannery and Horner 1994; Schmidt et al. 2000; Schreibman et al. 2000; Singer et al. 1987; Tustin 1995). For example, Tustin (1995) compared the effects of antecedent notifications of a transition without notice (i.e., no warning signal) on the stereotypic behavior of an adult participant with ASD at a worksite using an $\mathrm{ABAB}$ design. The work supervisor functioned as the intervention implementer (training data were not presented for the implementer) for all sessions. In the baseline immediate change (i.e., no notice) condition, the worksite supervisor approached the participant with materials for the next task and asked him if he would like to complete the next task. Materials were immediately placed in front of the participant and completion was praised. During the advanced notice intervention condition, the worksite supervisor approached the participant and asked him if he would like to start the next task in $2 \mathrm{~min}$. The tasks were not placed immediately in front of the participant, rather they were placed on a table nearby and engagement in the next task was verbally praised. The data indicated a decrease in stereotypic behavior during phases where advanced notice was delivered prior to a transition. 


\section{Consequence-Based Strategies for Challenging Behavior}

Five studies addressed challenging behavior in the context of transitions using consequence-based strategies (Kern and Vorndran 2000; McCord et al. 2001; Repp and Karsh 1994; Waters et al. 2009). For example, McCord et al. (2001) implemented a treatment package that consisted of differential reinforcement of alternative behavior, extinction, and response blocking to decrease the self-injurious behavior (SIB) of two adults with profound intellectual disability admitted to a residential treatment center. A multiple baseline across settings design was used for one participant and a reversal design was used for the other. A trained therapist functioned as the implementer for all functional analysis, baseline, and treatment sessions. Before intervention, a modified functional analysis was conducted to determine the transition-related environmental variables maintaining the SIB. The assessment was used to determine whether a change in activity, the termination of an activity, a location change, or activity termination without a location change was maintaining SIB. For both participants, the function of their SIB was escape from a location change. During the treatment phase, participants' appropriate transition behavior was differentially reinforced (i.e., completion of transition or holding on to a wheel chair), and SIB was blocked and did not result in the termination of the transition. The data indicated a decrease in SIB associated with location change transitions across both participants. A decrease was also observed for one participant across settings.

Antecedent-Based Strategies for Teaching Transition-Related Behavior

Eleven studies were identified that outlined strategies for teaching transition-related behavior (Bryan and Gast 2000; Carson et al. 2008; Cihak 2011; Cihak et al. 2008, 2010; Connis 1979; MacDuff et al. 1993; Martin et al. 1987; Massey and Wheeler 2000; Mechling and Savidge 2010; Newman et al. 1995; Palmen et al. 2012; Sainato et al. 1987; Spriggs et al. 2007). For example, Cihak (2011) compared the effects of static picture cues to video modeling on the between-task transition behavior of four students with ASD in a selfcontained classroom using an alternating treatment design. The classroom teacher functioned as the interventionist for all baseline and intervention sessions. During the picture cue condition, students were presented with five pictures that depicted the completion of specific activities. The teacher delivered a prompt to check their schedule, and the students were required to locate their schedule and begin the next task. During the video modeling condition, the same prompt was delivered before task initiation, but the students were then required to activate video clips that depicted them completing the next scheduled task. For two students, mastery criterion was met faster with static prompts. The remaining two participants met mastery criterion more efficiently with video modeling.

Consequence-Based Strategies for Teaching

Transition-Related Behavior

Four studies were identified that implemented consequencebased strategies to teach transition skills (Carden-Smith and Fowler 1984; Connell et al. 1993a, b; Sowers et al. 1980). For example, Carden-Smith and Fowler (1984) examined the effects of a token economy on the transition behavior of six children with language and developmental delays in a classroom setting. Three students were chosen to participate in experiment 1, and an ABAC design was used. During intervention, each student in the classroom was assigned to a team and was awarded points for independent participation in transitions (e.g., walking to the restroom). Each transition chosen for intervention was worth one point, and accumulation of points corresponded with different privileges during their daily outdoor activity period. During intervention, an increase in independent transitioning across students and settings was reported and maintained when peers were introduced as monitors. In experiment 2 , the effects of peer monitoring with and without teacher feedback were examined with the other three participants using a multiple baseline across participants design. A token system was implemented as outlined above. In one phase, the teacher delivered specific feedback to the peer monitors and ensured that points awarded were for the appropriate transition behavior. In the subsequent phase, teacher monitoring was withdrawn. The data from experiment 2 validated the previous findings that a token economy was effective in teaching and maintaining independent transition behavior with peers as the monitor.

\section{Discussion}

\section{Comments on Overall Effectiveness}

The current literature contains a variety of procedures for teaching independent transitioning skills and for decreasing challenging behavior related to between-task transitions. All of the studies reported positive results, and these interpretations were supported by a high PND for the majority of the studies. In a minority of the studies, although the authors noted positive results, these interpretations were not supported by a PND that was correlated with a high degree of effectiveness. A lack of correspondence between PND and author interpretation of the data was found in four studies (Banda and Kubina 2006; Palmen et al. 2012; Flannery and Horner 1994; Sowers et al. 1980). For example, Banda and Kubina (2006) cited positive results in decreasing noncompliance to 
transition with one student with ASD. During intervention, a high-probability request sequence was implemented prior to each transition. The data indicated a slight decrease in noncompliance $(M=2.7 \mathrm{~min}$; range $2.1-3.7 \mathrm{~min})$ in the first withdrawal phase and a larger, but still relatively small decrease in noncompliance in the second withdrawal phase $(M=$ 2.31; range 1.5-2.7 $\mathrm{min}$ ). Although a decrease was noted, the data were variable and a large number of data points between baseline and intervention overlapped. Using the criterion outlined by Scruggs and Mastropieri (1998), the overlap observed in the Banda and Kubina (2006) would indicate that the procedure was ineffective. Although only three studies were found in the current search, the discrepancies between author findings and PND may highlight a major limitation in the PND metric. Specifically, PND may be highly insensitive to instances where changes in trend do not coincide with changes in level. In the study of Banda and Kubina (2006), this limitation may be evident. During the first intervention phase, the data were stable and no change in level or trend was observed. In the second intervention phase, a clear change in trend in the desired direction can be observed without a change in level. From the data presented, the intervention demonstrated an effect on challenging behavior, but using PND, this improvement in the target response was not as significant.

A second general finding was that the most used interventions, and teaching procedures were antecedent-based, which may indicate that they are more effective or potentially easier to implement. Across both categories, antecedent-based methods were used in $77 \%$ of the studies. Overall, antecedent-based strategies were no more effective than consequence-based strategies, except in studies where teaching independent transitioning was the dependent variable. In these studies, antecedent-based strategies were more successful than consequence-based strategies.

Lastly, less than half of the reported studies $(n=13)$ documented measures of maintenance $(n=14)$ and/or generalization $(n=9)$. For many skills, maintenance and generalization may be required for the student to be competent in multiple environments. Transitions from task to task or location to location occur frequently across school and community settings (Carta et al. 1990). Maintenance of transitioning skills and the generality of those skills outside of teaching conditions may be required for an individual to be fully independent. Although limited, all of the studies in which generalization and maintenance was assessed demonstrated successful skill generalization (e.g., Carson et al. 2008) and maintained performance was reported (e.g., Massey and Wheeler 2000).

Maintenance of observed gains was demonstrated between five sessions (Newman et al. 1995; Schmidt et al. 2000) and 1 year (Repp and Karsh 1994). For example, Repp and Karsh (1994) implemented a differential reinforcement of alternative behavior procedure to decrease the occurrence of escape- maintained challenging behavior during transitions. During intervention, a transition that occurred without the occurrence of challenging behavior was verbally praised. Reinforcement was systematically faded throughout the intervention from every $5 \mathrm{~s}$ to every minute. The data indicated a marked change in challenging behavior in relation to baseline and these results maintained at much lower levels at a 1-year follow-up. Overall, many of the studies that reported maintenance focused on relatively short-term demonstrations of maintained gains (e.g., sessions).

Measures of generalization were more prevalent than maintenance, but were still somewhat limited. The majority of the studies that reported a measure of generalization ( $86 \%$ ) only reported the effects of the intervention generalizing to a novel setting. Although these results are promising, the small number of reports of generalization (22\%) from this literature and the limited ways generalization was demonstrated indicate future investigations are necessary. As stated previously, transitioning occurs across environments, with different stimuli, and across significant others. For example, an individual who engages in challenging behavior should have an intervention that is effective at both home and school, in the presence of a variety of individuals, and with a variety of stimuli present.

\section{Functional Behavior Assessment}

As stated above, less than half of the total studies that targeted challenging behavior ( $46 \%$ ) implemented a functional behavior assessment (FBA) or functional analysis (FA) prior to intervention. Functional assessments, specifically FAs, have been demonstrated to be useful in a variety of settings (Hanley 2012; Hanley et al. 2003). Several researchers have supported the inclusion of an FBA when constructing behavioral supports for individuals with disabilities (O'Neill et al. 1997). Yet in this review, the inclusion of an FBA or FA did not increase the probability of a successful intervention. FAs and FBAs are typically conducted when the goal of the intervention is to target the maintaining variable (i.e., source of reinforcement). Although this is one benefit of a functional assessment, it is not the only information that is acquired. Functional assessments also can yield information that lead to determining relevant discriminative stimuli and motivating operations precede a response, which could be useful in constructing effective antecedent-based interventions (Hanley et al. 2003; Miltenberger 2006).

The probability of identifying an appropriate intervention for challenging behavior did not seem to be impacted by the inclusion of an FBA or FA for either antecedent- or consequence-based interventions. For example, Schreibman et al. (2000) did not assess for potential functions of challenging behavior with one student with ASD. The absence of information regarding behavior function did not impact the 
effectiveness of the video priming intervention implemented. The overall finding that FBA or FA information did not impact intervention effectiveness is similar to the findings of Gresham et al. (2004). In their review of school-based intervention studies, Gresham et al. (2004) found that interventions that were based on FBAs or FAs were no more effective than those where the assessments were not conducted. It should be noted that these data may be somewhat misrepresentative of what occurs in natural contexts or outside of controlled experiments. Gresham et al. (2004) surveyed peer-reviewed journals, which tend to publish positive outcomes. Nevertheless, in the current review, the presence of a functional assessment was not correlated with more positive results.

With the limited implementation of functional assessments in the current review, it seems that challenging behavior during transitions functions to escape a demand context (e.g., Davis et al. 1998) or to gain access to a previous set of stimulus conditions (e.g., Waters et al. 2009). Similarly, the antecedent conditions that may occasion problem behavior during transitions may be the onset of those conditions (e.g., teacher walking to student with a worksheet). From this information, it may be possible to tentatively make two general conclusions about FBAs and FAs in the context of transitionrelated challenging behavior. First, in general, individuals may engage in challenging behavior to escape a demand context brought on by the transition or to gain access to previous environmental stimulation (Flannery and Horner 1994). Second, from the current literature, it appears to be possible to create interventions that are as effective as those that are created using FBA or FA information. Taken together, these two points may indicate that a clinician may be able to implement an effective intervention to decrease transitionrelated challenging behavior in many cases without implementing an FBA or FA. In cases where the target behavior does not respond to an empirically based intervention (e.g., picture schedule, differential reinforcement of alternative behavior), an FBA or FA may be required to construct an effective intervention.

\section{Implementers and Settings}

All individuals transition between places and activities a number of times each day. For example, a schoolteacher may begin their workday by transitioning from their car in the school parking lot to their classroom. The typical day for individuals with disabilities is no different. They may be required to engage in a number of transitions throughout a typical day; to facilitate these transitions, a number of implementers may be required. In the current review, the majority of implementers were classroom teachers and vocational workers. Because of the high percentage of the day transitioning (Carta et al. 1990; Schmidt et al. 2000), classroom or vocational staff may be the individuals who most often report observing transition difficulties.

Although transition difficulties may occur in classroom or vocational settings, the ability to transition independently may be essential for success in other environments. For example, transitioning from watching television to cooking dinner may be one skill needed for an individual with an intellectual disability to live with minimal supports. These types of transitions may require demonstrations provided by significant others outside of work or school settings. In the current review, caregivers or parents comprised $6 \%$ of the implementers reported. Similarly, interventions were conducted in community settings (e.g., homes) in $28 \%$ of the studies. Although independent transitioning may generalize outside of the initial context, it would be beneficial for individuals outside of school environments to provide further teaching when necessary. Additionally, some transition-related problems that need intervention may not be replicated fully in a school or clinic context. Further implementation in community settings with parents as the interventionist may be necessary to create more robust programming for transition skills.

\section{Limitations}

Percent Nonoverlapping Data In the current review, PND was used to quantify the results observed in each study. Although this metric is the most widely used method of objectively determining intervention effectiveness in singlesubject research (Parker et al. 2011), Wolery et al. (2010) cite several limitations of PND that should be taken into account when viewing the current results. First, PND may be impacted by the presence of variability and trend in the baseline condition (Ma 2006). For example, Kern and Vorndran (2000) investigated the effects of preferred activities and adult interaction on flopping to the floor in the context of transitions. During baseline, a low degree of variability was observed, but these data overlapped with data gathered during intervention yielding a PND that did not correspond to a high degree with graphic representation. This study illustrates the impact that low levels of variability may have on the PND of an effective intervention.

Second, ceiling and floor effects may increase the probability of low PND scores. This limitation is especially significant for studies where the research questions were to increase a target behavior to $100 \%$ or decrease a target behavior to $0 \%$. Ceiling and floor effects were accounted for by adjusting the protocol using the suggestions by Didden et al. (1997). Although not present in the current review, this adjustment may not be possible for phases where a small number of data points are collected. For example, if the intervention phase were shorter than the baseline phase, deleting data from the PND analysis would not be possible. 
Inclusion Criteria The current review only surveyed articles that were published in peer-reviewed English language journals were included. These specific criteria may have resulted in the exclusion of a number of studies (Schlosser et al. 2007). First, we only included peer-reviewed literature and excluded studies that may be considered "gray" literature (e.g., dissertations, theses, etc.) Although a limitation to this specific review, "gray" literature may not be appropriate for inclusion in systematic reviews because of the lack of stringent peer review.

Second, only articles written in English were included. In many literature reviews, this may be an inherent limitation because of author language abilities and resources. Although most likely a limitation in the majority of literature reviews published in English language journals, the probability that effective interventions were not identified because of publication in a foreign language journal should still be stated.

Study Design Constraints In the current review, only studies with single-case designs (SCD) were chosen. For the specific population targeted (e.g., people with ASD), single-case design is the experimental methodology most often implemented, as many behavioral excesses or deficits are very idiosyncratic and in some cases may not be appropriate for study using group designs. Although choosing SCD as the sole experimental design included, the larger number of studies identified may be indicative of robust literature using SCD as an experimental methodology.

Search Terms The purpose of all literature reviews is to identify the relevant information for review in a given base of studies (Schlosser et al. 2007). In the current review, search terms were carefully selected based on commonly used terms that may appear as keywords in article abstracts. Many of these terms come from either contact with education or behavior analytic literature, and other fields (e.g., speech language pathology, occupational therapy) may use different, but similar terms to describe procedures for intervention with transition difficulties. Because of this potential disparity, some search terms may have been inadvertently omitted.

\section{Future Research}

In the current review, a number of intervention strategies were effective for increasing independent transitioning skills (e.g., picture schedules) and decreasing challenging behavior (e.g., DRA). Although the research supports these interventions, several lines of future inquiry may be suggested. First, the use of an FBA or FA as a formal assessment protocol to analyze transitions does not seem to be fully supported by the current literature. In this review, interventions that were based on an FBA or FA were no more effective than interventions where these data were not collected (e.g., Cale et al.
2009). This does not indicate that FBA or FA are not useful in determining behavioral function, although this literature indicates that when transition difficulties are the target behavior, a set of empirically supported procedures (e.g., activity schedules) may be an effective first course of action. Future research may focus of further investigating this finding by directly comparing interventions developed based on information from pre-intervention functional assessments and those that are not.

Second, the reason for the effectiveness of antecedentbased interventions is relatively unknown for both reducing challenging behavior and teaching independent transitioning skills. Few studies have fully investigated the antecedent variables that may promote independent transitions (e.g., Waters et al. 2009). Flannery and Horner (1994) suggested that the use of antecedent-based strategies may increase the predictability of a student's environment, which may introduce more salient discriminative stimuli that signal transitions. For students who do not engage in independent transitioning, antecedent-based strategies may introduce additional discriminative stimuli into the environment that increase the probability of an independent transition. In the case of students who engage in challenging behavior, antecedent-based strategies may lessen the aversiveness of transitions. Future research is needed to determine the mechanisms underlying these results and provide further evidence in relation to the effectiveness of antecedent-based strategies.

Third, maintenance and generalization were demonstrated in less than half of the reviewed studies. Transition skills are required in multiple environments and are ubiquitous in educational settings (Carta et al. 1990). Transition skills that are learned in one setting may not be useful if they do not generalize to novel settings or maintain over time. Future research should document data related to both maintenance and generalization and present the strategies that were used to increase the occurrence of these outcomes. A large proportion of these studies were conducted in environments that employed qualified professionals as the implementers. Transitions occur in a variety of settings with a number of individuals, and demonstrations of success in alternate environments are needed. For example, some students may engage in challenging behavior in both home and school settings. Intervening in one setting does not necessarily mean that benefits will carry over to another setting with an untrained implementer. Future studies should investigate methods of intervening in settings outside of controlled settings (e.g., community settings) and with less qualified interventionists (e.g., parents).

Finally, PND was the metric used to quantify the results. Although PND is the most widely used metric for quantifying single-subject research, Wolery et al. (2010) note some potentially significant limitations. One of the most significant limitations is the insensitivity of PND to changes in data trends. For the current review, studies were classified as positive 
based on author report. In some instances, positive outcomes via author report did not agree with the PND score. In these cases, an observable change in the target response was observed, but not to a level where a high PND was calculated. Future investigations should determine the extent to which PND verifies the outcomes of visual inspection. For the current review, the PND score should be viewed as a compliment to author report.

\section{Conclusion}

In the current review, 32 studies were examined. All of the studies reviewed reported positive results. The majority of these results were supported by PND scores in the effective range (i.e., $70-100 \%$ ). Positive results were also observed across disability categories and ages. Overall, there were a number of effective strategies identified for both teaching independent transition skills and intervening when challenging behavior occurred in the context of a transition. The results from this review provide four general conclusions.

First, antecedent-based interventions for both challenging behavior and for teaching independent transition behavior were the most prevalent. Second, antecedentbased strategies were more effective in teaching independent transition skills, but they were equally as effective as consequence-based strategies for decreasing challenging behavior. Third, antecedent-based strategies were effective in both teaching transitions and decreasing challenging behavior, though the reasons for the positive effects have not been empirically demonstrated. Lastly, the reports of generalization and maintenance, although encouraging, are limited. Transitions occur constantly and generality of those skills may be needed for an individual to be competent in a given environment. Those skills should also be maintained over time to ensure continued success.

\section{References}

Arlin, H. A. (1979). Teacher transitions can disrupt time flow in classrooms. American Educational Research Journal, 16, 42-56.

Banda, D. R., \& Kubina, R. M. (2006). The effects of a high-probability request sequence technique in enhancing transition behaviors. Education and Treatment of Children, 29, 507-516.

Bryan, L. C., \& Gast, D. L. (2000). Teaching on-task and on-schedule behaviors to high-functioning children with autism via picture activity schedules. Journal of Autism and Developmental Disorders, 30, 552-567.

Cale, S. I., Carr, E. G., Blakely-Smith, A., \& Owen-DeSchryver, J. S. (2009). Context-based assessment and intervention for problem behavior in children with autism spectrum disorder. Behavior Modification, 33, 707-742.
Carden-Smith, L. K., \& Fowler, S. A. (1984). Positive peer pressure: the effects of peer monitoring on children's disruptive behavior. Journal of Applied Behavior Analysis, 17, 213-227.

Carson, K. D., Gast, D. L., \& Ayres, K. M. (2008). Effects of photo activity schedule book on independent task changes by students with intellectual disabilities in community job sites. European Journal of Special Needs Education, 23, 269-279.

Carta, J. J., Atwater, J. B., Schwarts, I. S., \& Miller, P. A. (1990). Applications of ecobehavioral analysis to the study of transitions across early childhood settings. Education and Treatment of Children, 13, 298-315.

Cihak, D. F. (2011). Comparing pictorial and video modeling activity schedules during transitions for students with autism spectrum disorders. Research in Autism Spectrum Disorders, $5,433-441$.

Cihak, D. F., Kessler, K., \& Alberto, P. A. (2008). Use of handheld prompting system to transition independently through vocational tasks for students with moderate and severe intellectual disabilities. Education and Training in Developmental Disabilities, 43, 102110.

Cihak, D. F., Fahrenkorg, C., Ayres, K. M., \& Smith, C. (2010). The use of video modeling via a video iPod and a system of least prompts to improve transitional behaviors for students with autism spectrum disorders in the general education classroom. Journal of Positive Behavior Interventions, 12, 103-115.

Connell, M. C., Carta, J. J., \& Baer, D. M. (1993a). Programming generalization of in-class transition skills: teaching preschoolers with developmental delays to self-assess and recruit contingent teacher praise. Journal of Applied Behavior Analysis, 26, 345-352.

Connell, M. C., Randall, C., Wilson, J., Lutz, S., \& Lamb, D. R. (1993b). Building independence during in-class transitions: teaching in-class transition skills to preschoolers with developmental delays through choral-response-based self-assessment and contingent praise. Education and Treatment of Children, 16, 160-174.

Connis, R. T. (1979). The effects of sequential pictorial cues, self-recording, and praise on the job task sequencing of retarded adults. Journal of Applied Behavior Analysis, 12, 355-361.

Cooper, J. O., Heron, T. E., \& Heward, W. L. (2009). Applied behavior analysis (2nd ed.). Upper Saddle River: Pearson.

Davis, C. A., Reichle, J. E., \& Southard, K. L. (1998). High-probability requests and a preferred item as a distracter: increasing successful transitions in children with behavior problems. Education and Treatment of Children, 23, 423-440.

Dettmer, S., Simpson, R. L., Smith-Myles, B., \& Ganz, J. B. (2000). The use of visual supports to facilitate transitions of students with autism. Focus on Autism and Other Developmental Disabilities, 15, 163169.

Didden, R., Duker, P. C., \& Korzilius, H. (1997). Meta-analytic study on treatment effectiveness for problem behaviors with individuals who have mental retardation. American Journal on Mental Retardation, 101, 387-399.

Dooley, P., Wilczenski, F. L., \& Torem, C. (2001). Using an activity schedule to smooth school transitions. Journal of Positive Behavior Interventions, 3, 57-61.

Flannery, K. B., \& Horner, R. H. (1994). The relationship between predictability and problem behavior for students with severe disabilities. Journal of Behavioral Education, 4, 157-176.

Gresham, F. M., McIntyre, L. L., Olson-Tinker, H., Dolstra, L., McLaughlin, V., \& Van, M. (2004). Relevance of functional behavioral assessment research for school-based interventions and positive behavioral support. Research in Developmental Disabilities, 25, 19-37.

Hanley, G. P. (2012). Functional assessment of problem behavior: dispelling myths, overcoming implementation obstacles, and developing new lore. Behavior Analysis in Practice, 5, 54-72. 
Hanley, G. P., Iwata, B. A., \& McCord, B. E. (2003). Functional analysis of problem behavior: a review. Journal of Applied Behavior Analysis, 36, 147-185.

Kern, L., \& Vorndran, C. M. (2000). Functional assessment and intervention for transition difficulties. Journal of the Association of Persons With Severe Handicaps, 25, 212-216.

Ma, H. H. (2006). An alternative method for quantitative synthesis of single-subject research: percentage of data points exceeding the median. Behavior Modification, 30, 598-617.

MacDuff, G. S., Krantz, P. J., \& McClannahan, L. E. (1993). Teaching children with autism to use photographic activity schedules: maintenance and generalization of complex response chains. Journal of Applied Behavior Analysis, 26, 89-97.

Martin, J. E., Elias-Burger, S., \& Mithaug, D. E. (1987). Acquisition and maintenance of time-based task change sequence. Education and Training in Mental Retardation, 22, 250-255.

Massey, N. G., \& Wheeler, J. J. (2000). Acquisition and generalization of activity schedules and their effects on task engagement in a young child with autism in an inclusive pre-school classroom. Education and Training in Mental Retardation and Developmental Disabilities, 35, 326-335.

McCord, B. E., Thompson, R. J., \& Iwata, B. A. (2001). Functional analysis and treatment of self-injury associated with transitions. Journal of Applied Behavior Analysis, 34, 195-210.

Mechling, L. C., \& Savidge, E. J. (2010). Using a personal digital assistant to increase completion of novel tasks and independent transitioning by students with autism spectrum disorder. Journal of Autism and Developmental Disorders, 41, 687-704.

Miltenberger, R. G. (2006). Antecedent interventions for challenging behaviors maintained by escape from instructional activities. In J. K. Luiselli (Ed.), Antecedent assessment \& intervention (pp. 101124). Baltimore: Paul H. Brookes Publishing.

Newman, B., Buffington, D. M., O’Grady, M. A., McDonald, M. E., Poulson, C. L., \& Hemmes, N. S. (1995). Self-management of schedule following in three teenagers with autism. Behavioral Disorders, 20, 190-196.

O’Neill, R. E., Horner, R. H., Albin, R. W., Sprague, J. R., Storey, K., \& Newton, J. S. (1997). Functional assessment and program development for problem behavior: a practical handbook. Pacific Grove: Brooks.

Palmen, A., Didden, R., \& Verhoeven, L. (2012). A personal digital assistant for improving independent transitioning in adolescents with high-functioning autism spectrum disorder. Developmental Neurorehabilitation, 15, 401-413.

Parker, R. I., Vannes, K. J., \& Davis, J. L. (2011). Effect size in singlecase research: a review of nine nonoverlap techniques. Behavior Modification, 35, 303-322.

Repp, A. C., \& Karsh, K. G. (1994). Hypothesis-based interventions for tantrum behaviors of persons with developmental disabilities in school settings. Journal of Applied Behavior Analysis, 27, 21-31.
Rule, S., Fiechtl, B. J., \& Innocenti, M. S. (1990). Preparation for transition to mainstreamed post-preschool environments: development of a survival skills curriculum. Topics in Early Childhood Special Education, 9, 78-90.

Sainato, D. M., Strain, P. S., Lefebvre, D., \& Rapp, N. (1987). Facilitating transition times with handicapped preschool children: a comparison between peer-mediated and antecedent prompt procedures. Journal of Applied Behavior Analysis, 20, 285-291.

Schlosser, R. W., Wendt, O., \& Sigafoos. (2007). Not all systematic reviews are created equal: considerations for appraisal. EvidenceBased Communication Assessment and Intervention, 1, 138-150.

Schmidt, J., Alper, S., Raschke, D., \& Ryner, D. (2000). Effects of using a photographic cueing package during routine school transitions with a child who has autism. Mental Retardation, 38, 131-137.

Schreibman, L., Whalen, C., \& Stahmer, A. C. (2000). The use of video prompting to reduce disruptive transition behavior in children with autism. Journal of Positive Behavior Interventions, 2, 3-11.

Scruggs, T. E., \& Mastropieri, M. A. (1998). Summarizing single-subject research: issues and applications. Behavior Modification, 22, 221242.

Scruggs, T. E., Mastropieri, M. A., \& Castro, G. (1987). The quantitative synthesis of single-subject research: methodology and validation. Remedial and Special Education, 8, 24-33.

Singer, G. H., Singer, J., \& Horner, R. H. (1987). Using pretask requests to increase the probability of compliance for students with severe disabilities. Journal of the Association for Persons with Severe Handicaps, 12, 287-291.

Sowers, J., Rusch, F. R., Connis, R. T., \& Cummings, L. E. (1980). Teaching mentally retarded adults to time-manage in a vocational setting. Journal of Applied Behavior Analysis, 13, 119-128.

Spriggs, A. D., Gast, D. L., \& Ayres, K. M. (2007). Using picture activity schedule books to increase on-schedule and on-task behaviors. Education and Training in Developmental Disabilities, 42, 209 223.

Sterling-Turner, H. E., \& Jordan, S. S. (2007). Interventions addressing transition difficulties for individuals with autism. Psychology in the Schools, 44, 681-690.

Tustin, D. (1995). The effects of advance notice of activity transitions on stereotypic behavior. Journal of Applied Behavior Analysis, 28, 9192.

Waters, M. B., Lerman, D. C., \& Hovanetz, A. N. (2009). Separate and combined effects of visual schedules and extinction plus differential reinforcement on problem behavior occasioned by transitions. Journal of Applied Behavior Analysis, 42, 309-313.

Wilder, D. A., Chen, L., Atwell, J., Pritchard, J., \& Weinstein, P. (2006). Brief functional analysis and treatment of tantrums associated with transitions in preschool children. Journal of Applied Behavior Analysis, 39, 103-107.

Wolery, M., Busick, M., Reichow, B., \& Barton, E. E. (2010). Comparison of overlap methods for quantitatively synthesizing single-subject data. The Journal of Special Education, 44, 18-28. 